\title{
Time to eliminate LNT: The NRC needs to adopt LT and eliminate ALARA
}

\author{
Siegel JA ${ }^{1}$, Sacks $B^{2}$, Welsh JS ${ }^{3 *}$ \\ ${ }^{1}$ Nuclear Physics Enterprises, Marlton, NJ, USA \\ ${ }^{2} \mathrm{US}$ FDA (retired, diagnostic radiologist), Green Valley, AZ, USA \\ ${ }^{3}$ Dept of Radiation Oncology, Stritch School of Medicine Loyola University- Chicago, Maywood, IL, USA
}

\begin{abstract}
The Linear No-Threshold (LNT) model has been the basis of radiation regulatory policy for more than 70 years but, lacking valid scientific foundation, remains only an assumption. The LNT extrapolation from observed high-dose effects to putative low-dose responses entails that all ionizing radiation exposure down to zero is harmful proportionally to dose; this implicitly denies the existence of a protective biological response to the observed initial radiation-induced damage. Denying such a response, LNT further implies that the harm is cumulative throughout life, regardless of how low the dose or dose rate. But there are evolved protective biological responses that quickly repair or remove any radiogenic damage from low doses and dose rates, thereby preventing damage from accumulating. LNT and its offspring, ALARA (as low as reasonably achievable), do not err on the side of caution and are not conservative. Instead the public needs protection from radiophobia rather than from low-dose/rate radiation exposure. The NRC and other regulatory agencies should no longer base their radiation protection standards on LNT. Instead regulatory policy should be based on a linear (down to a) threshold (LT) model.
\end{abstract}

\section{Introduction}

The Linear No-Threshold (LNT) model has been applied to lowdose and low-dose-rate ionizing radiation for more than 70 years, but it lacks valid scientific foundation and so remains only an assumption. Nonetheless, this assumption remains the orthodox foundation of radiation protection science and forms the basis of radiation regulations and public policy. That is, radiation protection policies are dominated by a paradigm based on an assumption without empirical foundation. Radiophobia, promoted by use of LNT (and ALARA), has prompted three petitions for rulemaking (PRMs) to the U.S. Nuclear Regulatory Commission (NRC) on 23 June 2015 (80 FR 35870), requesting that it cease using this model in favor of a model indicating radiationinduced benefit at low doses (i.e., hormesis). The NRC requested public comments and that its Advisory Committee on the Medical Uses of Isotopes (ACMUI) consider the requests in the PRMs. In its Final Report dated October 28, 2015, the ACMUI, after considering the petitions, made the following recommendation to the Commission (emphasis added):

The "correct" dose-response model for radiation carcinogenesis remains an unsettled scientific question. There is a large, and growing, body of scientific literature as well as mechanistic considerations which suggest that 1) the LNT model may overstate the carcinogenic risk of radiation at diagnostic medical, occupational, and environmental doses and 2) such low doses may, in fact, exert a hormetic (i.e., a beneficial or protective) effect. However, in the absence of definitive refutation of the LNT model and while strongly encouraging continued investigation critically comparing alternative models, regulatory authorities should exercise prudent (though not excessive) conservatism in formulating radiation protection standards. The ACMUI therefore recommends that, for the time being and subject to reconsideration as additional scientific evidence becomes available, the NRC continue to base the formulation of radiation protection standards on the LNT model.

Unfortunately, while the ACMUI acknowledged the vast and evergrowing body of scientific literature refuting the Linear No-Threshold (LNT) model - often also affirming hormesis - it nevertheless recommended that the NRC maintain its reliance on the LNT model, because it would be the "prudent" and "conservative" regulatory strategy to take. The ACMUI apparently believes that LNT modelderived regulations and policies, since they assume the maximum harm, are protective of public health. But the aftermath of the Chernobyl and Fukushima nuclear accidents demonstrate the falsity of this assumption.

In fact, overestimated risk has devastated hundreds of thousands of lives, many shortened thereby. Furthermore, the radiophobia promoted by the LNT-derived "as low as reasonably achievable" (ALARA) principle (a regulatory requirement that was not specifically addressed in the ACMUI report) is firmly entrenched in medical radiological imaging. This one-sided assumption of radiogenic damage without biological response blinds radiologists and patients to actual risks, such as misdiagnosis due to nondiagnostic (underexposed) images or avoidance of medical imaging. Additionally, it obscures and denies demonstrable potential health benefits from the radiation itself,

Correspondence to: Welsh JS, Director of Clinical \& Translational Research Department of Radiation Oncology Stritch School of Medicine Loyola University- Chicago, USA, Tel: 708-216-2561; E-mail: shermanwelsh@ gmail.com

Key words: Linear no-threshold model, ALARA, low-dose radiation exposure risk, adaptive response, Nuclear Regulatory Commission, radiation protection

Received: December 18, 2016; Accepted: January 12, 2017; Published: January 16,2017 
thus compounding the error. While demanding "definitive" refutation of LNT before regulatory policy is changed, the ACMUI leaves unspecified what they would consider definitive refutation. Thereby they reserve the sole right to decide what evidence would suffice as refutation, all but guaranteeing that the current policy remains in place indefinitely.

Interestingly, there has never been any "definitive" or undisputable evidence supporting LNT. In contrast, there is "definitive" evidence that its use has resulted in harmful, even deadly, consequences. There are countless scientists whose performance, or at least acknowledgment, of voluminous experimental and observational studies already convinces them that the refutation has been definitive. This is the point that lies at the crux of our argument. The ACMUI misassigns the burden of proof. Rather than those with the preponderance of evidence bearing the burden, it should rightly be borne by those who have none that their data actually support. The ACMUI seems to be shirking its responsibility by awaiting "definitive" refutation from the putative "mainstream" of expert scientific opinion, such as represented by scientific committees like NCRP, BEIR, and ICRP. Such change will not likely come soon, since these committees are comprised of significant overlapping members. The recommendations from these groups have led to a longterm, mutually-reinforcing "scientific consensus" that has utterly failed the public and has produced far more harm than good, by repeatedly defending and promoting the fictitious LNT model. Many people have died as a result of unjustified and unsupported policies formulated on the basis of LNT, so there is great urgency in terminating regulatory use of LNT and its corollary the ALARA principle.

LNT advocates have made scientific claims pertaining to lowdose radiation exposure that have proven to be false. One is that lowdose radiogenic up-regulation of protective adaptive responses only partially offsets the carcinogenic damage. Another is the self-justifying admission that epidemiological studies cannot detect the risk because of the noisy background $[1,2]$. When it serves their interests, however, Little, for one, has reversed himself, unashamedly asserting that certain recent large epidemiological studies by the International Agency for Research on Cancer (IARC) demonstrate that low-dose/rate radiation increases cancer mortality-but only by $0.1 \%$ over a baseline risk of some $25 \%$ [3]. Little thus defends two incompatible claims: first, that epidemiological studies cannot provide evidence for LNT because of statistical invisibility, and, second, that if some how they appear to provide this evidence, then they should be uncritically accepted as having, in fact, accomplished the impossible. But the appearance of success in the IARC studies is based on circular reasoning, as well as on illegitimate statistical maneuvering that satisfies a desire for mathematical convenience, as we (BS and JAS) have shown, thereby resolving the apparent paradox [4]. Considerations of biology and physics should be the source and/or final arbiter of scientific hypotheses about ionizing radiation, and not assumptions that happen to yield mathematically convenient relationships. If epidemiological studies indicate no significant radiation-related excess cancer at low doses $(<100-200 \mathrm{mSv})$, it is not simply because the expected effect is too small to be detected. Statistical invisibility is a straw-man argument that applies only to LNT, since, rather than being invisible, the increased cancer risk is nonexistent, or, more precisely, is actually negative (hormetic) - as hundreds of studies have demonstrated with statistical significance. That is why evidence for hormesis is, in fact, statistically visible, and that is why it is necessarily ignored by LNT proponents in their determination to preserve the demonstrably false LNT assumption as the basis of policy [5].

\section{NRC should eliminate LNT and ALARA and adopt a Linear Threshold (LT) model}

The LNT assumption derives from incomplete, early-20th-century genetic experimental observations of mutations in fruit flies. It was a result of extrapolation far below the experimental range, an a priori unjustified maneuver that the data, furthermore, suggested was not even accurate [6]. Experimenter Hermann Muller, in his 1946 Nobel Lecture, asserted unequivocally that there is no threshold for harm all the way down to zero dose (or dose rate), despite the fact that the data only extended from high doses down to 4,000 mGy and from high dose rates down to $0.1 \mathrm{mGy} / \mathrm{min}$-making his claim a stunning nonsequitur. In the 2-3 years following his Nobel Lecture, experimental data obtained at doses down to $250 \mathrm{mGy}$ and dose rates as low as 0.0174 $\mathrm{mGy} / \mathrm{min}$ suggested a Linear Threshold (LT) - i.e., linear down to a threshold-relationship between mutation frequency and radiation dose. Furthermore, contrary to the interpretation of the experimenters, their data suggested a marked dose rate effect at this dose threshold [6]. Ten years later the NAS BEAR committee extended this initial error from fruit flies to humans and applied it to carcinogenesis and birth defects in subsequent generations. So, there has been no evidence, "definitive" or otherwise, supporting the LNT model, nor has any evidence since then ever validated the carcinogenicity of low-dose radiation exposure. On the contrary, countless experimental and observational studies show that such doses do not cause cancer, but more likely help prevent it, through its stimulation of several layers of evolved biological defence mechanisms.

The assumed LNT extrapolation from high-dose to low-dose responses entails not only that all ionizing radiation exposure down to zero is harmful, proportional to dose, but also entails the denial that there is any adaptive biological response at all to the (undisputed) initial radiation-induced damage. It is also taken to mean that radiation yields cumulative harm throughout life, regardless of how low the dose or dose rate. Both claims are demonstrably false, since there is an evolved protective biological response, and any dose rate low enough to allow that response to take place permits remediation of any damage that may be done in intact organisms by either repair or, that failing, removal of the damaged cells by apoptosis or the immune system. Thus, LNT has led to regulations and policies that are not merely unneeded for protection but, on the contrary, turn out in practice to produce great harm [7-9]. For example, more than 1,600 deaths resulted after the Fukushima nuclear accident from the misguided LNT-based evacuation policy, both in the immediate aftermath and from its being kept in force for many years.

Astonishingly, the atomic-bomb survivor cohort of the Life Span Study (LSS), the single most important dataset for estimating radiation effects in humans after acute high dose-rate exposures, is consistent with at least a LT dose-response relationship, if not a hormetic relationship $[8,9]$. Yet the regulatory basis of radiation "protection" remains firmly wedded to the LNT fiction and its consequently unjustifiable ALARA policy. The bottom line is that neither LNT nor ALARA errs, in fact, on the side of caution, but rather errs firmly on the side of harm.

It is also noteworthy that the spontaneous endogenous mutation rate - due to reactive oxygen species (ROS) produced by normal metabolism-is roughly a million-fold greater than that due to low-doserate radiation. The minuscule ratio of radiogenic-to-nonradiogenic (endogenous) DNA damage increases at much higher doses and/or 
dose rates, but it remains small for the radiation exposures that would be encountered by the public from either medical imaging or nuclear accidents, or even by radiation workers [10].

Different mechanisms operate at high and low doses delivered acutely. At very high doses delivered at sufficiently high dose rates, the body is indeed unable to repair or eliminate all the damage done, whereas the biological responses at low doses or low dose rates more than serve to overcome any damage. These include DNA repair (at the molecular level), apoptosis and bystander/rescue effects (on the cellular level), antioxidant production (on the tissue level), and immunological removal of surviving damaged cells (on the organismal level). These layers of adaptive responses collaborate for the harmonized function of all organisms and involve more than 150 genes [8,11-14]. If there is any characteristic feature of healthy living organisms, it is the naturally selected ability to respond to damage in order to preserve homeostasis. Had such biological responses not evolved, endogenous DNA damage alone would cause us to develop cancer every minute of the day-even if the earth lacked natural radiation from ground and sky.

Recent research shows the incompleteness of mechanistic models of radiation-induced cancer that imply that, in a linear fashion, DNA double-strand breaks (DSBs) necessarily lead to unrepaired chromosome aberrations resulting in cancer. These inadequacies indicate that the various intervening biological response mechanisms (at the molecular, cellular, tissue, and organismal levels) are necessary to complete the picture. Regardless of whether initial low-dose DNA damage itself is linear, taken together with the body's defensive response the net result is nonlinear [12]. This is true whether the low dose is delivered acutely, as evidenced by the LSS cohort, or, even more so, when radiation is delivered on a protracted, low-dose-rate basis.

\section{Radiation damage is not cumulative}

The assertion that radiation damage is cumulative, no matter the dose or dose rate-a logical corollary of the fiction-is directly contradicted by the experience with fractionation of high-dose radiation therapy, in which recovery of normal tissue occurs between treatments [15]. Additionally, low dose radiation stimulates repair or removal of damage over and above the immediate radiogenic damage and therefore provides enhanced protection against additional damage over time, including damage from subsequent higher radiation exposures, endogenous production of reactive oxygen species, and other non-radiogenic damage. The net result is reduction of damage below baseline levels, likely contributing to a lifetime-cancer-risk reduction and increased longevity-the essence of hormesis.

The BEIR VII report grants the existence of DNA repair but pronounces it "incomplete." And because the BEIR committee assumes, without evidence, imperfect repair of initial DNA damage, the report dismisses a dose threshold for carcinogenicity, thereby completely ignoring the additional higher-level mechanisms of defense against radiation-caused damage in those cells in which DNA repair does fail [16]. In fact, the report asserts that evidence of such defense mechanisms (i.e., hormetic effects) is weak and contradicted by direct measures of incomplete DNA repair at low doses, based on citing, but incorrectly characterizing the experimental results of, a paper by Rothkamm and Löbrich [17].

Starkly contradicting BEIR VII's characterization, this paper provides evidence for mechanisms reducing both spontaneous and radiation-induced damage below baseline levels (a hormetic effect). Rothkamm and Löbrich found that DSBs induced in cultures (in vitro) of nondividing primary human fibroblasts by very low radiation doses ( $1 \mathrm{mGy}$ ) remain unrepaired for many days, in contrast to more efficient DSB repair that was observed at somewhat higher, yet still low, doses. But, as they further found, the level of DSBs in irradiated cultures decreases to that of unirradiated cell cultures if the cells are allowed to proliferate after irradiation, as would occur in vivo. Thus, they found evidence of either repair or cell-destroying and organism-preserving apoptosis-a finding disturbingly omitted in the BEIR VII report [4]. Crucially, cell cultures lack all those mechanisms that occur at higher levels in intact organisms, including tissue and immune responses. BEIR VII's misquoting from their own chosen reference raises serious questions about the integrity of at least some of its authors and the validity of the entire report - which constitutes a major pillar of LNT advocacy.

DSB repair occurs after CT scans, which involves low doses, albeit at relatively high dose rates. Interestingly, in contrast to certain results obtained in vitro, normal individuals after undergoing CT examinations repaired initial DSBs, observable minutes after exposure, to background levels within 24 hours [18]. That is, in vivo repair kinetics do not indicate a compromised repair capacity for the radiationinduced DSB damage expected to occur at these low radiation doses, even when delivered at relatively high dose rates.

These in vivo repair measurements, however, uncovered a DSB repair defect in one person who had previously shown exceptionally severe side effects after undergoing radiotherapy for rectal cancer. The authors concluded that the clinical radio sensitivity of this patient was likely caused by a DSB repair defect- i.e., a hyporepairability defect. But, as we have reported, it is likely that a minority of individuals are "hyporepairable" and may have a higher-than-average low-dose threshold that must be exceeded to more fully activate their protective systems [19].

In any event, the adaptive response in mammalian cells and mammals due to low-dose radiation exposure likely operates within a certain window that is delimited by both upper and lower dose thresholds, typically between about 1 and 100 mGy [20]. Essentially a hormetic range, a phenomenon found not only in radiation exposure, but also in response to any and all physical and chemical stressors [19]. There are generally three stressor-specific ranges of response: too little, just right (hormetic range), and too much. These dose thresholds will vary somewhat from person to person, being dependent on a number of parameters, including such things as dose rate, genetic characteristics (e.g., genetic variations in TP53), age, tissue type, and medical condition. Exposures above the upper threshold are generally detrimental, while exposures below the lower threshold may be less protective against, or even contributory to, disease risk. That is, rather than all radiation being harmful, we all require a certain minimum exposure to radiation for optimal health, rendering the ALARA concept not merely useless but actually harmful. Cells that remain damaged due to hyporepairability are most likely eliminated by apoptosis, a beneficial effect resulting in a dose-response relationship certainly below the LNT prediction of cancer, and likely below "no effect" - a subject for further research $[21,22]$.

Another study, involving interventional cardiologists (exposed to a median of $4 \mathrm{mSv} / \mathrm{y}$ ), compared them with unexposed controls (who were likely still exposed to at least comparable natural background radiation dose rates). Low-dose, chronic exposure was associated with two adaptive cellular responses: enhanced antioxidant defense and increased apoptotic response [23]. These likely compensate for 
increased ROS production and contribute to maintaining cellular homeostasis. An accompanying editorial noted that these data confirm low-dose protective responses [24].

Additional low-dose radiation has also been shown to stimulate the immune system, causing a reduction in cancer rates below background [25]. While residents in areas with higher natural background radiation levels ( $3.3 \mathrm{mSv} / \mathrm{y}$ ) were found to have higher frequencies of chromosome aberrations than lower-background control populations $(1.1 \mathrm{mSv} / \mathrm{y})$, importantly they had lower all-cancer mortality, indicating both that the frequency of chromosomal aberrations should not be used as a surrogate for cancer mortality and that observations of initial damage should be followed longer to more meaningful endpoints [26]. This strongly argues against the LNT model of radiation-induced cancer.

\section{Conclusion}

\section{Eliminate LNT/ALARA and adopt LT}

LNT and its offspring ALARA are fatally flawed, focusing only on molecular damage, while ignoring organismal protective molecular, cellular, tissue and organismal level biological responses that repair or remove the damage. At low radiation doses and/or dose rates protective adaptive responses more than compensate for the damage, repairing or removing some of the far greater damage caused by endogenous processes $[4,8,14]$. With no evidence supporting the LNT assumption, and much evidence to support LT or even hormesis, LNT advocates are responsible, perhaps unwittingly, for promoting radiophobia with all its negative consequences. Most importantly, and in contrast to what its proponents assert, LNT and its offspring, ALARA, do not err on the side of caution and are not conservative; therefore, no policy based on these demonstrably false foundations can be protective.

The sources of radiophobia can no longer be ignored: low-dose radiation exposure has no documented pathway to harm, whereas LNT and ALARA decidedly do [9]. The public requires protection from radiophobia rather than from radiation. It is illusory to believe that adherence to ALARA will convince the public that radiologists are protecting them from harm, when it is ALARA that convinces them that there is something to fear. Regulatory adherence to LNT and ALARA must therefore end.

Contrary to ACMUI's recommendation to the Commission, the NRC should no longer continue to base its radiation protection standards on the LNT assumption, pending that never-to-arrive day when "definitive" contrary evidence becomes available. Such scientific evidence is already available to "definitively" refute LNT. Regulatory policy should instead be based on a linear (down to a) threshold (LT) model, the actual model suggested even by the data gathered by Muller and colleagues before and during the 1940s and a model consistent with the more recent interpretations of the atomic bomb survivor dataset. An LT model partially retains linearity, confirming a linear dose-response relationship due to high dose exposure, but only down to a threshold of $100 \mathrm{mGy}$ or possibly even higher (to be determined by further research), below which there is either no risk or an actual health benefit for the vast majority of people. There may be a small number of individuals in this zero-to-threshold dose range who are hyporepairable relative to the majority of the population and thus will exhibit more damage at lowest doses, but it remains to be seen whether their other levels of response-apoptosis, bystander/rescue effects, and immunological-compensate for this defect. An LT model could be used for dose management in order to set regulatory dose limits and for dose assessment down to the threshold dose level. If an LT model were to be implemented-a decision that stands to save many lives-the useless and harmful ALARA policy would naturally be obviated.

\section{References}

1. Doss M, Little MP, Orton CG (2014) Point/counterpoint: low-dose radiation is beneficial, not harmful. Med Phys 41: 070601. [Crossref]

2. Little MP, Wakeford R, Tawn EJ, Bouffler SD, de Gonzalez AB (2009) Risks associated with low doses and low dose rates of ionizing radiation: why linearity may be (almost) the best we can do. Radiol 251:6-12. [Crossref]

3. Little MP (2015) Ionising radiation in the workplace. BMJ 351: h505.

4. Sacks B, Meyerson G, Siegel JA (2016) Epidemiology without biology: false paradigms, unfounded assumptions, and specious statistics in radiation science (with commentaries by Inge Schmitz-Feuerhake and Christopher Busby and a reply by the authors). Biol Theory 11: 69-101. [crossref]

5. Siegel JA, Sacks B, Stabin MG (2015) LNT 999. Health Physics News XLIII: 23-24.

6. Siegel JA, Pennington CW, Sacks B, Welsh JS (2015) The birth of the illegitimate linear no-threshold model: an invalid paradigm for estimating risk following low-dose radiation exposure. Am J Clin Oncol. [Crossref]

7. Siegel JA, Stabin MG (2012) RADAR commentary: use of linear no-threshold hypothesis in radiation protection regulation in the United States. Health Phys 102 90-99. [Crossref]

8. Siegel JA, Welsh JS (2016) Does imaging technology cause cancer? Debunking the linear no-threshold model of radiation carcinogenesis. Technol Cancer Res Treat 15 249-256. [Crossref]

9. Siegel JA, Pennington CW, Sacks B (2016) Subjecting radiological imaging to the linear no-threshold hypothesis: a non sequitur of non-trivial proportion. $J$ Nucl Med 58: 1-6. [Crossref]

10. Billen D (1990) Spontaneous DNA damage and its significance for the "negligible dose" controversy in radiation protection. Radiat Res 124: 242-45. [Crossref]

11. Pateras IS, Havaki S, Nikitopoulou X, Vougas K, Townsend PA, et al. (2015) The DNA damage response and immune signaling alliance: is it good or bad? Nature decides when and where. Pharmacology \& Therapeutics 154: 36-56. [Crossref]

12. Brooks AL, Dauer LT (2014) Advances in radiation biology: effect on nuclear medicine. Semin Nucl Med 44: 179-186. [Crossref]

13. Luckey TD (1991) Radiation Hormesis. Dose Response 4: 169-190. [Crossref]

14. Feinendegen LE, Pollycove M, Neumann RD (2009) Low-dose cancer risk modeling must recognize up-regulation of protection. Dose-Response 8: 227-252. [Crossref]

15. Taylor LS (1980) Some nonscientific influences on radiation protection standards and practice: the 1980 Sievert lecture. Health Phys 39: 851-874. [Crossref]

16. National Research Council of the National Academies (2006) Health Risks from Exposure to Low Levels of Ionizing Radiation: BEIR VII Phase 2. Washington, DC: The National Academies Press.

17. Rothkamm K, Löbrich M (2003) Evidence for a lack of DNA double-strand break repair in human cells exposed to very low x-ray doses. Proc Natl Acad Sci USA 100 5057-5062. [Crossref]

18. Löbrich M, Rief N, Kühne M (2005) In vivo formation and repair of DNA double strand breaks after computed tomography examinations. Proc Natl Acad Sci USA 102: 8984-8989. [Crossref]

19. Siegel JA, Marcus CS, Welsh JS, Pennington CW, Stabin MG (2016) Regulatory application of the LNT hypothesis and ALARA to protect radiosensitive people is misguided. Health Physics News XLIV: 23-24.

20. Mitchel REJ (2010) The dose window for radiation-induced protective adaptive responses. Dose-Response 8: 192-208. [Crossref]

21. Dauer LT, Brooks AL, Hoel DG. Morgan WF, Stram D, et al. (2010) Review and evaluation of updated research on the health effects associated with low-dose ionising radiation. Radiat Prot Dosimetry 140: 103-136. [Crossref]

22. Bauer G (2007) Low dose radiation and intercellular induction of apoptosis: potentia implications for the control of oncogenesis. Int J Radiat Biol 83: 873-888. [Crossref]

23. Russo GL, Tedesco I, Russo M (2012) Cellular adaptive response to chronic radiation exposure in interventional cardiologists. Eur Heart J 33: 408-414. [Crossref]

24. Gori T, Münzel T (2012) Biological effects of low-dose radiation: of harm and hormesis. Eur Heart J 33: 292-295. [Crossref] 
Welsh JS (2017) Time to eliminate LNT: The NRC needs to adopt LT and eliminate ALARA

25. Liu SZ (2006) Cancer control related to stimulation of immunity by low-dose radiation. Dose Response 5: 39-47. [Crossref]
26. Chen D, Wei L (1991) Chromosome aberration, cancer mortality and hormetic phenomena among inhabitants in areas of high background radiation in China. $J$ Radiat Res 2(suppl 2): 46-53. [Crossref]

Copyright: (C2017 Welsh JS. This is an open-access article distributed under the terms of the Creative Commons Attribution License, which permits unrestricted use, distribution, and reproduction in any medium, provided the original author and source are credited. 